\title{
CARACTERÍSTICAS DE LA DOCENCIA TRANSDISCIPLINARIA: DESARROLLO DE INSTRUMENTOS PARA EVALUARLA
}

\section{Characteristics of Transdisciplinary Teaching: Development of Instruments to Evaluate it}

\author{
Mabel Artidiello ${ }^{1}$, María Elena Córdoba ${ }^{2}$ y Lucero Arboleda ${ }^{3}$
}

Recibido :20-12-2016• Aprobado: 8-5-2017

\begin{abstract}
Resumen
Este trabajo proporciona una propuesta con los componentes para el abordaje y la evaluación de la docencia transdisciplinaria. Desde una profunda reflexión teórica de la transdisciplinariedad, se analizaron sus principios y fundamentos, a fin de identificar las características esenciales que la determinan. Los componentes identificados, organizados en categorías y subcategorías fueron descritos y sometidos a revisión por expertos en materia transdisciplinar; posteriormente, se realizó un taller de formador de formadores para docentes de las cinco áreas académicas del INTEC y una prueba piloto. A partir de las experiencias mencionadas y de la validación externa de las características para este tipo de docencia, se procedió a diseñar una herramienta de evaluación capaz de contener tanto las categorías como las subcategorías identificadas, así como también sus descriptores. Para tal metodología de evaluación, y luego de analizar diversas técnicas y herramientas, se optó por una rúbrica construida con base en las características descritas inicialmente, por considerarla una poderosa herramienta de evaluación, la cual constituye además un elemento fundamental para el fortalecimiento de este tipo de docencia, fungiendo como un mecanismo de formación docente.
\end{abstract}

Palabras clave: transdisciplinariedad; docencia transdisciplinaria; metodología de evaluación; herramienta de evaluación; rúbrica.

1. Profesora investigadora del Instituto Tecnológico de Santo Domingo (INTEC), República Dominicana. Correo electrónico: mabel.artidiello@intec.edu.do

2. Profesora investigadora del Instituto Tecnológico de Santo Domingo (INTEC), República Dominicana

\begin{abstract}
This paper provides a proposal with the components for the approach and evaluation of transdisciplinary teaching. From a deep theoretical reflection of transdisciplinarity, its principles and foundations were analyzed in orderto identify the essential characteristics that determine it. The identified components, organized into categories and sub-categories, were described and reviewed by transdisciplinary experts, followed by a teacher-training workshop, for teachers in the five academic areas of INTEC and a pilot test. Based on the abovementioned experiences, and the external validation of the characteristics for this type of teaching, an evaluation tool capable of containing both the categories and subcategories identified, as well as their descriptors was designed. For this methodology of evaluation and after analyzing different techniques and tools, a rubric was chosen based on the characteristics described initially, considering it a powerful evaluation tool, which is also a fundamental element for the strengthening of this type of teaching, and represent a mechanism of teacher training.
\end{abstract}

Key words: transdisciplinarity; transdisciplinary teaching; evaluation methodology; evaluation tool; rubric.

3. Directora de la Biblioteca del Instituto Tecnológico de Santo Domingo (INTEC), República Dominicana. Correo electrónico: lucero.arboleda@intec.edu.do 


\section{Introducción}

El estudio inició con una profunda revisión bibliográfica que tuvo como resultado la identificación de un acervo documental, con datos de gran valor para los estudiosos de la transdisciplinariedad.

Esta investigación hizo importantes aportes e impactó en la práctica docente de los participantes debido a las impresiones que de ellos hemos recogido $y$, adicionalmente, estos a su vez están resultando multiplicadores de la reflexión para una docencia innovadora, integradora, y con características que podemos identificar como transdisciplinarias. Así mismo contar con una herramienta evaluativa para una docencia en la cual en muchas Instituciones de Educación Superior (IES) no tenemos experiencia, resulta de gran valor porque permitirá a estas instituciones, así como al cuerpo docente, tener claras algunas características propias de este tipo de docencia, por lo que su utilización será en sí misma un mecanismo útil para la formación docente.

\section{Conceptualizaciones sobre transdisciplinariedad, docencia y evaluación}

\section{Transdisciplinariedad}

La aparición de la palabra transdisciplinariedad, conceptualmente sustentada, es difícil de ubicar en el tiempo. No obstante, según Bourguignon (1997), fue Jean Piaget quien, en 1970, en el marco de un coloquio sobre interdisciplinariedad, manifestó: "En fin, a la etapa de las relaciones interdisciplinares, puede esperarse que le suceda una etapa superior que sería 'transdisciplinar', que no se contentaría con alcanzar interacciones o reciprocidades entre investigaciones especializadas, sino que situaría estos vínculos al interior de un sistema total sin fronteras estables entre las disciplinas" (pp. 9-10).

En el plano de la investigación y de la educación, ambas con palmarias demostraciones de agotamiento del enfoque disciplinar, la transdisciplinariedad sugiere, entre muchos otros aportes, aquellos orientados a Sistemas de Organización del Conocimiento (SOC) que, con respecto a la investigación, sean coherentes con sus tres pilares fundamentales: niveles de la realidad, lógica del tercero incluido, y complejidad. Con relación a la educación, los SOC deben expresar los alcances de la transdisciplinariedad, la cual aporta significativas diferencias que van desde un modelo de fertilización del conocimiento in vitro, hacia un rico modelo de fertilización in vivo que cuenta con el aporte de todos sus actores; desde un solo nivel de realidad, hacia varios niveles de la realidad.

Esta búsqueda también puso de manifiesto el hecho de que el término transdisciplinariedad no ha sido dotado de una estructura para entrar a los SOC con nombre propio. Así lo revelan los resultados de la consulta, tanto de bases de datos multidisciplinarias, como especializadas, auxiliada por herramientas de búsqueda de diferente índole, incluidas las herramientas de descubrimiento, donde para ubicar trabajos sobre transdisciplinariedad debimos hacerlo bajo simples palabras clave o descriptores muy generales.

En el tesauro de Unesco no existe el término investigación transdisciplinaria. Ausencia que nos permite aventurarnos a justipreciar los retos conceptuales que plantea la transdisciplinariedad y que, indiscutiblemente, tienen impacto en un sistema de gestión de conocimiento que exhibe arritmia y gran rezago con relación a la dinámica de la transdisciplinariedad, la cual según muchos autores es un "movimiento" intelectual y académico que, por su gran apertura, es mucho más amplio y receptivo que una "escuela" ideológica con reglas fijas de pensamiento. Movimiento apuntalado, sobre todo, por la Unesco y por el Centro Internacional de Investigaciones y Estudios Transdisciplinarios (CIRET).

No obstante, más allá de reclamar la estructuración de un corpus terminológico, la transdisciplinariedad 
nos está planteando la situación de que los temas se clasifican en conformidad con la terminología especializada de las disciplinas que los estudian. Esto significa que los investigadores con perspectiva transdisciplinar no disponen de clasificaciones que les permitan recuperar con sentido de pertinencia, calidad y oportunidad la información requerida, propiciando entonces el grave riesgo de la innecesaria duplicación de esfuerzos. En general, esta situación afecta a quienes desarrollan investigaciones académicas que conllevan la aplicación de una o más teorías y métodos al estudio de uno o más fenómenos. Todos ellos, aunque ubicados en disciplinas distintas, deben ser buscados a través de sistemas unidisciplinarios.

Por lo tanto, aunque contamos con herramientas que, como los tesauros, se proponen controlar el vocabulario y limitar el sentido de los términos para facilitar la recuperación por la semejanza entre petición y representación o para sugerir términos de búsqueda, tal como antes señalamos, en ellos brilla por su ausencia el término transdisciplinariedad.

Aun con las limitaciones antes señaladas, pudimos encontrar que, a pesar de que se admite que el año 1970 marca la aparición de la palabra transdisciplinariedad, al mover hacia atrás la línea del tiempo, encontramos dicha palabra como parte del título de documentos que circulan a través de las numerosas bases de datos académicas, es posible encontrarla desde 1947:

Autor: Erich Jantsch.

Título: inter- and transdisciplinary university: a systems approach to education and innovation.

Fuente: Higher Education Quarterly, Vol. 1 Issue 1, p7-37 (31 p.), Feb. 1947.

Estos comentarios y su correspondiente ejemplo, nos permiten señalar que plantear el tema de la transdisciplinariedad en el contexto de los Sistemas de Organización del Conocimiento (SOC) representa un paso de avance hacia la inclusión de la transdisciplinariedad en herramientas cognitivamente ergonómicas que permitan organizar la información; esto en principio a través de tesauros, y luego a través de herramientas más sofisticadas que está llamada a proveer la web semántica, para que podamos contar con dinámicas herramientas de descubrimiento de información, capaces de asumir los nuevos retos que plantea el cambio en sus diferentes dimensiones: material, mental, espiritual y social.

\section{Docencia y transdisciplinariedad}

En el plano de la educación, un plan de estudios transdisciplinario sobrepasa el enfoque multidisciplinario porque su propósito es integrar varias disciplinas y saberes no académicos para lograr crear un plan a partir de la experiencia en lo que se busca enseñar. De este modo, la educación transdisciplinaria implica el completo involucramiento de los estudiantes, de manera tal que intervenga la participación de todas las dimensiones del ser humano, que posibiliten un aprendizaje a través de sus experiencias y de la reflexión, de manera integral.

Ahora bien, hacer la transición del enfoque disciplinario al enfoque transdisciplinario requiere de un modelo que tienda a la reflexión en y sobre la práctica, y a una cooperativa de producción de saber que trasciende el modelo de la racionalidad técnica (Galvani, 2006; Schön, 2006; Pineau, 2006, 2007, citados por Espinosa, 2010). La formación aún está basada en las disciplinas y en la especialización. Se necesita un cambio de paradigma que recupere la integralidad del saber, donde todas las disciplinas propias de una experiencia interactúen.

La implementación del enfoque transdisciplinario y su complejidad destaca cambios en los procesos formativos y en las personas involucradas en ellos. El aprendizaje presenta cambios significativos en el campo del conocimiento; estos cambios implican 
aprender a pensar complejamente la realidad a través de herramientas nuevas, así como saber los pilares transdisciplinares y los principios complejos (Nicolescu, 1998; Morin, 2005, citados por Espinosa, 2010).

El Centro Internacional para los Estudios Transdisciplinarios (CIRET) planteó en 1987 los siguientes propósitos de la transdisciplinariedad:

- Combatir la segmentación del conocimiento

- Reducir la brecha entre la investigación y la toma de decisión concerniente a la vida social

- Estudiar la similitud entre distintas ramas del conocimiento (Cassinari, et al., 2011).

Ciencia, educación, y sociedad, conjuntamente con la innovación, deben formar un sistema integral para dirigir la sociedad desde la universidad, donde inter y transdisciplinariedad se conviertan en elementos operativos para el funcionamiento del sistema (Espinosa, 2010). Una vez más es evidente cómo la transdisciplinariedad se traduce en la unión de elementos que permiten una mejor comprensión y dirección de las situaciones humanas y sociales, en esta ocasión introduciendo a la universidad como el espacio donde se desarrolla esta danza entre ciencia y sociedad.

Para Nicolescu (1998): “El pensamiento fragmentado es incompatible con la búsqueda de la paz sobre esta Tierra. La emergencia de una cultura y una educación para la paz reclama una evolución transdisciplinaria de la educación y, particularmente de la Universidad" (p. 114). El autor con este planteamiento introduce la cultura de paz como resultado directo de la evolución de la perspectiva transdisciplinaria de la educación, señalando específicamente la educación superior. Desde su perspectiva no es posible alcanzar la paz bajo la concepción fragmentada del mundo y el modo de conocerlo. De allí que declara la educación superior transdisciplinaria como la generadora de la paz sobre la tierra.

\section{Evaluación de la docencia}

La educación ha cambiado, pero también la evaluación cambió. Al preguntarnos para qué evaluar la docencia, Robalino y Corner (2006) hacen una reflexión en la que indican que la evaluación tiene como finalidad básica mejorar la educación a través de la optimización de la calidad de la enseñanza, para alcanzar niveles más altos de idoneidad profesional y ampliar la comprensión de sí mismos, de los papeles que juegan y de los contextos. Esto es posible si enmarcamos la evaluación en una concepción amplia y abarcadora, cuyo fin es favorecer el aprendizaje y cuyo accionar se debe desarrollar como un proceso continuo.

Hay muchos estudiosos de la educación que refuerzan esta postura y han realizado aportes que la enriquecen. Caballero, et al. (2014) plantean que la actividad de evaluación debe ser una función que forma parte de la mejora continua. Por otro lado, la evaluación de la docencia universitaria debería ser una representación equitativa y precisa del desempeño docente, una vía para el mejoramiento de la educación superior. (Ramírez \& Montoya, 2014, p. 81)

En ese mismo orden, podemos identificar que la evaluación ya no cumple una función de medir conocimientos, sino de apoyar el aprendizaje y ser un elemento de mejora para las IES. Así lo corrobaran Stake, Contreras \& Arbesú (2011): "la tarea de evaluar la docencia se convierte en una necesidad actual, no sólo para fines de medición de la calidad, sino como parte intrínseca de un proceso de mejora necesario de la institución universitaria”.

Por tanto, en la concepción de la educación actual, se entiende la evaluación como el instrumento indispensable en el proceso enseñanza aprendizaje, para la mejora institucional, para el aprendizaje del estudiantado y para la formación del cuerpo docente, quienes gracias a ella pueden tener una mirada de lo que hacen y de cómo lo hacen, además de 
reflexionar sobre el proceso, entenderlo y mejorarlo constantemente.

La evaluación, en sí misma, ha de ser un espacio de reflexión y de mejora de la realidad, pero su oportunidad y sentido de repercusión, tanto en la personalidad del evaluado como en su entorno y en el equipo del que forma parte, ha de ser entendida y situada adecuadamente para posibilitar el avance profesional de los docentes (Tejedor, 2012, p. 322). De allí la importancia de diseñar instrumentos de evaluación idóneos para cada modalidad docente.

\section{Metodología}

El estudio inicialmente se concibió de tipo descriptivo y durante su desarrollo se definió como de asociación. Una vez descritas las características de la docencia transdisciplinaria, se pretendió demostrar que esta docencia requiere instrumentos de evaluación diseñados especialmente para ella, ya que con los usados en otras modalidades docentes no se lograría tal evaluación. Partimos del supuesto de que la docencia transdisciplinaria posibilita abordar cualquier problemática o contenido de actualidad de una manera mucho más abarcadora que desde los otros enfoques. La hipótesis a defender fue la siguiente: La docencia transdisciplinaria es un proceso complejo con características propias que no pueden valorarse adecuadamente con los instrumentos tradicionales utilizados para evaluar la docencia.

El enfoque fue eminentemente cualitativo, aunque se realizó una agrupación de frecuencias en los resultados de las evaluaciones hechas en el taller y en la prueba piloto. La información recibió tratamiento de análisis del discurso, teoría fundamentada y utilización de Atlas.ti, con la intención de que este análisis permitiera la identificación de categorías y subcategorías que son la base de nuestros análisis y resultados presentados.
La propuesta metodológica consistió en una profunda revisión bibliográfica, la identificación de las características que debe tener la modalidad transdisciplinar en la docencia y la selección de una modalidad para evaluarla.

Posteriormente, se realizó la tipificación de los indicadores fundamentales y secundarios que caracterizan una docencia transdisciplinaria. El resultado obtenido se puso a consideración de dos expertos en transdisciplinariedad, esto con el objetivo de que validaran los indicadores identificados.

Luego, se impartió un taller presencial de formador de formadores con 12 participantes (dos estudiantes, un egresado y nueve docentes), con duración de un trimestre, y en el siguiente trimestre se llevó a cabo la prueba piloto en la que participaron 10 de los 12 que originalmente lo hicieron en el primer curso (un estudiante, un egresado y ocho docentes).

Debido a que el facilitador reside en Puerto Rico, el diseño instruccional estuvo dado por la transmisión vía Skype en tiempo real. Conformaron la prueba cuatro sesiones correspondientes a un curso regular de este profesor en una cátedra de Estudios Generales de la Universidad de Puerto Rico. Al final de cada clase, se habilitó un espacio, también vía Skype, con los participantes en esta propuesta, a fin de aclarar dudas, opinar y reflexionar sobre lo ocurrido en cada sesión.

Al final de toda la experiencia, el grupo completo de diez participantes mantuvo un encuentro presencial con el facilitador, que implicó debatir y analizar opiniones y dudas. Además de estar convencidos de las bondades de esta modalidad pedagógica, se llegó a la conclusión general de que es necesario seguir profundizando y socializando estas experiencias con el propósito de contar con docentes empoderados de prácticas pedagógicas capaces de trascender las fronteras disciplinares y construir conocimientos. 
Al finalizar la experiencia, a los actores involucrados se les entregó una rúbrica de evaluación, con el fin de recoger desde su perspectiva una valoración de la experiencia.

Después de estas dos experiencias, consideramos indispensable construir una herramienta especialmente diseñada para evaluar este tipo de docencia, debido a la importancia de las implicaciones de la evaluación de esta modalidad pedagógica.

\section{Resultados}

\section{Componentes que identifican a una docencia como transdisciplinar}

Una vez realizada la revisión bibliográfica, nos dimos a la tarea de compilar los componentes que definen una docencia como transdisciplinaria, los cuales son el resultado de las revisiones de literatura, el análisis y la reflexión con respecto a lo que consideramos que debemos tomar en cuenta como elementos indispensables en una docencia transdisciplinaria. Este resultado de identificación de los componentes estuvo concluido luego de la contrastación que se llevó a cabo posteriormente.

A ambos expertos se les entregó el material resultante, al cual le hicieron importantes aportes que enriquecieron significativamente el documento. Luego, se cruzaron los comentarios de ambos a fin de que fueran validados por los dos. El resultado de esa etapa se presenta a continuación:

Con posterioridad al análisis, podemos evidenciar que las características identificadas en este estudio como necesarias para una docencia transdisciplinaria, si bien no podemos decir que sean las únicas, sí son un requisito clave para la misma. $\mathrm{Al}$ preguntarnos cuántas de estas deben evidenciarse y cuándo podemos decir que reúnen los requisitos para afirmar que cumple con cada una de las características en cuestión, partimos por retomar las características y la descripción que habíamos dado, con el comentario correspondiente.

\section{Características de una docencia transdisciplinaria}

Transgresora: esto debido a que implica la elaboración de ese nuevo cuerpo de saber que "atraviesa" a las disciplinas, lo que le da su especificidad característica a uno u otro campo transdisciplinar con relación a los otros. Todos son transdisciplinares, pero cada uno presenta su especificidad. De tal forma que trasciende las disciplinas, las valora, las complementa, las integra y logra ir más allá. Además, supera el ámbito de poder exclusivo de los "expertos" y desarrolla sensibilidad abierta a las diversas manifestaciones de la sabiduría. Va construyendo un nuevo cuerpo de saber transversal a esas disciplinas, tomando de ellas - por alejadas que parezcan estar unas de otras- nociones, conceptos, enfoques, etc.; cuerpo de saber transversal que "les devuelve" a esas disciplinas para que lo utilicen, pues la transdisciplina no sustituye ni elimina a las disciplinas, sino que las complementa y trasciende su enfoque y la mentalidad disciplinar.

Cumplir con esta característica implica que, en la práctica docente, al integrar diversas disciplinas, voces y dimensiones, se logra trascender el enfoque de cada disciplina, elaborando un nuevo campo de saber que las incluye y las atraviesa.

Integradora de conocimientos: porque considera las diversas dimensiones de un problema o de una situación, partiendo de las áreas del saber o las disciplinas en que se manifiesta dicha docencia, pero sin restringirse a ellas, siempre reconociendo la estrecha relación que guardan unas con las otras. Es decir, están integradas en la realidad. Además, incorpora continuamente saberes y experiencias provenientes de actores situados en los más diversos lugares, no solo los que se producen en el mundo académico. Dando lugar a un "diálogo entre 
saberes" no solo disciplinares, sino entre el saber "experto" y el "lego", entre el occidental y todos aquellos que fueron negados, marginados e invisibilizados por la colonialidad (orientales, africanos, indoamericanos, etc.).

Cumplir con esta característica implica que cada situación particular es mirada desde múltiples dimensiones, entre las que se reflejen los saberes contextualizados provenientes de los mismos actores del problema, cada vez que sea posible. El rol docente, además, consiste en generar la problematización desde los vértices más variados, con el fin de que sean incorporados y cuestionados los diversos saberes que han sido convocados.

Democrática: pues los docentes y los estudiantes aprenden y construyen conocimientos por medio de una relación dialógica, en la que se valora significativamente la aportación de quienes participan del proceso. Al acercarse al conocimiento desde todas las aristas que puedan integrar, se trabaja de manera inclusiva, reconociendo que nadie tiene el monopolio del conocimiento, ni dentro ni fuera de la academia. También, es democrática porque pretende no dejar a nadie excluido, en el entendido de que la educación es un derecho. Por ello, es conveniente convertir el aula en una "comunidad de indagación".

Para cumplir con esta característica, la función docente es facilitar el diálogo, la deliberación de un intercambio colectivo entre educandos y docente. Y esa "dialógica" conjuga lo complementario con lo contradictorio, sustituye el "o esto o aquello" por "esto y aquello", o por "algo de esto y algo de aquello", en dependencia de las condiciones imperantes.

Inclusiva: todas las voces y experiencias deben ser incorporadas desde los diferentes tejidos que permean la realidad y a los cuales podemos acercarnos (espiritual, religioso, físico, cultural, científico, etc.). Se parte de aprovechar la multidimensionalidad de, prácticamente, cualquier problemática.
Cumplir con esta característica implica superar la convocatoria disciplinar al abordaje de cualquier problemática para incorporar además otras dimensiones que tradicionalmente no forman parte de la propuesta pedagógica, como el aspecto espiritual, religioso, oriental, africano, ritual, ancestral, etc.

Crítica: estimula el pensamiento, utiliza el cuestionamiento constante y es la principal herramienta para un aprendizaje generador de preguntas y respuestas que dan lugar a nuevos cuestionamientos. Se privilegia la pregunta como incitación al pensamiento y como gestora de respuestas. Se estimula al estudiante a producir preguntas que pueden quedar abiertas y sin respuestas inmediatas y mucho menos triviales, pues se reconoce la complejidad del pensamiento y la realidad de la que pretende dar cuenta. Se propicia el desarrollo de un pensamiento crítico, reflexivo, capaz de responder a las siete preguntas básicas: qué me dicen, quién(es) me lo dice, cómo me lo dicen, dónde me lo dicen, cuándo me lo dicen y, sobre todo, para qué y por qué me lo dicen.

Cumplir con esta característica implica que el docente propicia el cuestionamiento y la duda como forma de pensar respecto a cualquier elemento o problema que se esté analizando; una de las formas de hacerlo es mediante la utilización de la técnica de la pregunta. No solo pregunta el facilitador, sino que los estudiantes y el facilitador aprenden preguntando, cuestionando y poniendo en duda lo analizado, favoreciendo así el desarrollo de un pensamiento crítico.

Comprensiva: alcanza, penetra y contextualiza el conocimiento, reconoce la importancia de los contextos en los que se desarrolla la actividad educativa y aquellos donde viven tanto estudiantes como docentes. De ahí que se ubica en el aprendizaje que no extrapola el conocimiento para aprenderlo, sino que parte de su contexto real. Es conveniente vincular el tópico a tratar con circunstancias que sean cercanas a la vida y al contexto cotidiano -familiar, barrial, comunitario, citadino, nacional, etc. 
Para cumplir con esta característica el docente vinculará las problemáticas con los escenarios de la vida cotidiana que rodean la realidad de los participantes, pondrá en contexto la problemática y los actores que intentan un acercamiento a ella. El conocimiento situado o contextual deberá verse reflejado en una práctica docente transdisciplinaria.

Ética: el uso y la generación de conocimiento está al servicio de una inteligencia colectiva, entendida como aquella que permite la toma de decisiones social y éticamente sostenibles y robustas, que favorece a la mayoría en una sociedad.

El cumplimiento de esta característica se evidencia en toda la modalidad de conducción de la clase, cuando se observa un estilo honesto y transparente de integrar saberes, actores, voluntades, intereses, etc.; siempre privilegiando el bien común y entendiendo el conocimiento como patrimonio de la sociedad.

Adicionalmente a las características mencionadas como requisito para una docencia transdisciplinaria, las cuales se solapan frecuentemente, hay una cuya presencia es indispensable y que corresponde al facilitador que imparte docencia, más que a una característica de la misma. Sin embargo, en el análisis puede evidenciarse como indispensable, razón por la cual es necesario mencionarla: la humildad. No resulta aceptable entender una docencia transdisciplinaria con un docente que carezca de esta condición.
Consideramos que todas las características identificadas en este estudio deben verse reflejadas en una docencia transdisciplinaria. Quizá por la naturaleza del contenido o la temática, por momentos es posible que se manifieste más directamente una u otra, sin embargo, la ausencia de alguna de ellas podría estar reflejando un aspecto de mejora para esa práctica docente. Esta consideración podrá tener mayor soporte y sustento en la medida que se continúe con el análisis y el seguimiento de prácticas con intención de ser transdisciplinarias.

Por otra parte, la rúbrica de evaluación para la docencia transdisciplinar consideramos que aporta una herramienta valiosa cuyo propósito no será exclusivamente la evaluación, sino que resultará fundamental para la formación docente y para la mejora de este tipo de docencia.

\section{Rúbrica para evaluar la docencia transdisciplinaria}

Como elemento que resultó medular para este estudio, posteriormente a las experiencias de taller y la prueba piloto, consideramos indispensable generar un instrumento de evaluación para la docencia transdisciplinaria.

Elegimos como herramienta una rúbrica, la cual se construyó de acuerdo a las categorías y las dimensiones identificadas y que deberá ser validada en un próximo estudio. 


\begin{tabular}{|c|c|c|c|c|c|}
\hline I. Transgresora & $\begin{array}{c}\text { Excelente } \\
100-95\end{array}$ & $\begin{array}{c}\text { Bien } \\
94-85\end{array}$ & $\begin{array}{c}\text { Regular } \\
84-75\end{array}$ & $\begin{array}{c}\text { Deficiente } \\
74-65\end{array}$ & $\begin{array}{l}\text { Nulo } \\
64 \text { - } 0\end{array}$ \\
\hline $\begin{array}{l}\text { 1.1 Trasciende las disciplinas, las valora, las } \\
\text { complementa, las integra y logra ir más allá. } \\
\text { Además, transgrede el ámbito de poder exclu- } \\
\text { sivo de los "expertos" y desarrolla sensibilidad } \\
\text { abierta a las diversas manifestaciones de la sa- } \\
\text { biduría. } \\
\text { Trasciende el conocimiento básico y logra la } \\
\text { conexión con otras áreas y formas del saber que } \\
\text { no son parte del campo de estudio. }\end{array}$ & & & & & \\
\hline $\begin{array}{l}\text { 1.2 Se transgreden las fronteras disciplinarias, } \\
\text { los límites que configuran la identidad de las } \\
\text { disciplinas. Valora, complementa, integra y } \\
\text { proyecta el dominio del conocimiento sobre } \\
\text { diferentes disciplinas. } \\
\text { Emula realidades cotidianas y realiza interco- } \\
\text { nexiones de saberes con el diálogo de situacio- } \\
\text { nes similares y la discusión con diversidad de } \\
\text { puntos de vista, asumiendo como elemento de } \\
\text { rigor la inclusión del discurso del acervo po- } \\
\text { pular. }\end{array}$ & & & & & \\
\hline $\begin{array}{l}\text { 1.3 Se transgrede la epistemología occidental y } \\
\text { su canon -eurocéntrico-universalista, abriendo } \\
\text { el camino del pluralismo epistemológico. Invi- } \\
\text { ta a la discusión la diversidad de saberes ances- } \\
\text { trales, orientales, producto de la experiencia, } \\
\text { etc. } \\
\text { Respeta la confrontación de ideas, demostran- } \\
\text { do humildad, capacidad de escucha, sentido de } \\
\text { pertenencia, dominio ético y criticidad frente a } \\
\text { las ideas contradictorias o adversas con su pro- } \\
\text { pio criterio. }\end{array}$ & & & & & \\
\hline
\end{tabular}


2.4 Propicia el diálogo entre saberes, extrapolando a su propia práctica docente el conocimiento de diversos actores, provenientes de diversos lugares de procedencia, etnia, creencia o género, evidenciando la inclusión a múltiples sectores, considerando su opinión, respetando la diversidad y provocando la confrontación discursiva entre el saber científico, el saber académico y el no académico.

\begin{tabular}{|c|c|c|c|c|c|}
\hline I. Democrática & $\begin{array}{c}\text { Excelente } \\
100-95\end{array}$ & $\begin{array}{c}\text { Bien } \\
94-85\end{array}$ & $\begin{array}{c}\text { Regular } \\
84-75\end{array}$ & $\begin{array}{c}\text { Deficiente } \\
74-65\end{array}$ & $\begin{array}{l}\text { Nulo } \\
64-0\end{array}$ \\
\hline $\begin{array}{l}\text { 3.1 Es co-constructor de conocimiento, propi- } \\
\text { ciando que los estudiantes se empoderen de dicho } \\
\text { constructo mediante el diálogo participativo, el } \\
\text { respeto a la diversidad y la capacidad de escucha, } \\
\text { que quedan manifestados en las actividades de } \\
\text { interacción, el trabajo colaborativo y la reflexión } \\
\text { con los estudiantes, mostrando respeto y actitud } \\
\text { de escucha frente a las diversas opiniones y postu- } \\
\text { ras presentadas. }\end{array}$ & & & & & \\
\hline $\begin{array}{l}3.2 \text { Crea espacios para el trabajo inclusivo, permi- } \\
\text { tiendo la participación activa y el involucramiento } \\
\text { de los estudiantes, tomando en cuenta su opinión } \\
\text { y transformándolos en el centro de interés de los } \\
\text { procesos de aprendizaje, mediante la aplicación de } \\
\text { estrategias problematizadoras, proyectos, estudios } \\
\text { de caso y trabajo colaborativo. Al mismo tiempo, } \\
\text { evita la monopolización del conocimiento, trans- } \\
\text { formando a sus alumnos en hacedores de su pro- } \\
\text { pio aprendizaje, mediante la actitud de escucha y } \\
\text { reflexión frente a las situaciones del medio circun- } \\
\text { dante. }\end{array}$ & & & & & \\
\hline
\end{tabular}




\begin{tabular}{|c|c|c|c|c|c|}
\hline $\begin{array}{l}\text { 3.3 Transforma el aula en un laboratorio perma- } \\
\text { nente de investigación, en el cual, conjuntamente } \\
\text { con los estudiantes, construyen comunidades de } \\
\text { aprendizaje para la indagación y la constatación } \\
\text { de diversos saberes académicos, científicos y em- } \\
\text { píricos, en los que el docente y el estudiante en- } \\
\text { cuentran multiplicidad de oportunidades para el } \\
\text { desarrollo de sus áreas de conocimiento particular. } \\
\text { Lo que queda manifiesto mediante el diálogo y el } \\
\text { intercambio colectivo de ideas, manifestando dife- } \\
\text { rentes niveles de criticidad, mostrando actitud de } \\
\text { respeto y escucha. }\end{array}$ & & & & & \\
\hline $\begin{array}{l}\text { 3.4 Toma en consideración al estudiante y lo reco- } \\
\text { noce en sus potenciales, en sus valores y en lo po- } \\
\text { sitivo que tiene. Nunca le desacredita ni desprecia } \\
\text { su ignorancia, por el contrario, parte de ello para } \\
\text { tenderle retos de superación. Se preocupa por sa- } \\
\text { ber qué piensan sus estudiantes, qué sienten, qué } \\
\text { les preocupa o qué esperan. }\end{array}$ & & & & & \\
\hline IV. Inclusiva & $\begin{array}{c}\text { Excelente } \\
100-95 \\
\end{array}$ & $\begin{array}{c}\text { Bien } \\
94-85\end{array}$ & $\begin{array}{c}\text { Regular } \\
84-75\end{array}$ & $\begin{array}{c}\text { Deficiente } \\
74-65\end{array}$ & $\begin{array}{l}\text { Nulo } \\
64-0 \\
\end{array}$ \\
\hline $\begin{array}{l}\text { 4.1 Incorpora la diversidad de opiniones, expe- } \\
\text { riencias y posturas de los diferentes sectores que } \\
\text { conforman la realidad, y no sólo los que corres- } \\
\text { ponden al contexto académico, evidenciado me- } \\
\text { diante la inclusión del diálogo entre saberes, la } \\
\text { participación activa de los diferentes sectores, sus- } \\
\text { citando el encuentro entre el conocimiento empí- } \\
\text { rico, el académico y científico, mediante prácticas } \\
\text { integrales con los actores de la comunidad, pro- } \\
\text { yectos integrados para contrarrestar problemáticas } \\
\text { culturales, sociales y medio ambientales. }\end{array}$ & & & & & \\
\hline
\end{tabular}




\begin{tabular}{|l|l|l|l|l|}
\hline $\begin{array}{l}\text { 4.2 Impulsa la formación de habilidades críticas } \\
\text { y reflexivas para abordar cualquier problemática, } \\
\text { permitiendo la incorporación de otras propuestas } \\
\text { pedagógicas que no forman parte de la propuesta } \\
\text { pedagógica formal, tomando en cuenta el contexto } \\
\text { espiritual, religioso, desde lo cultural y ancestral. } \\
\text { Lo que queda evidenciado con la valorización del } \\
\text { acervo cultural, el análisis y la reflexión en su dis- } \\
\text { curso, la iniciativa de investigaciones en la acción } \\
\text { y las discusiones guiadas, rescatando la postura y la } \\
\text { opinión empírica de los diversos actores sociales, } \\
\text { sin implicar la etnia, la condición socioeconómica, } \\
\text { el nivel educativo o la perspectiva de género. }\end{array}$ & & & & \\
\hline $\begin{array}{l}\text { 4.3 Enriquece el acervo cultural mediático de la } \\
\text { cotidianidad social circundante, con la búsqueda } \\
\text { exhaustiva en diversas fuentes académicas y cien- } \\
\text { tíficas, que lo demuestra en la confrontación de } \\
\text { situaciones, habilidades discursivas, promoción } \\
\text { de técnicas y habilidades de estudios entre el sa- } \\
\text { ber conocido y el desconocido. Es capaz de emitir } \\
\text { conclusiones acertadas entre la diversidad de opi- } \\
\text { niones, a fin de plantear posibles soluciones a los } \\
\text { conflictos, no solo tangibles, sino también parti- } \\
\text { culares de los estudiantes y los actores insertos en } \\
\text { la comunidad. }\end{array}$ & & & & \\
\hline
\end{tabular}

\begin{tabular}{|c|c|c|c|c|c|}
\hline V. Crítica & $\begin{array}{c}\text { Excelente } \\
100-95\end{array}$ & $\begin{array}{c}\text { Bien } \\
94-85\end{array}$ & $\begin{array}{c}\text { Regular } \\
84-75\end{array}$ & $\begin{array}{c}\text { Deficiente } \\
74-65\end{array}$ & $\begin{array}{l}\text { Nulo } \\
64-0 \\
\end{array}$ \\
\hline $\begin{array}{l}\text { 5.1 Estimula el conocimiento mediante la formu- } \\
\text { lación constante de preguntas generadoras, susci- } \\
\text { tando experiencias de reflexión, evocación de si- } \\
\text { tuaciones de la realidad por la reflexión, el análisis } \\
\text { y la discusión entre docente y estudiantes. Esto se } \\
\text { evidencia en las exposiciones, las rutinas de clase, } \\
\text { las entrevistas y los grupos focales. }\end{array}$ & & & & & \\
\hline $\begin{array}{l}\text { 5.2 Propicia el desarrollo del pensamiento críti- } \\
\text { co con el cuestionamiento de diversas preguntas: } \\
\text { qué, quién (es), cómo, dónde, cuándo, para qué y } \\
\text { por qué; entre otras, mediante la elaboración de } \\
\text { guías de análisis. Y lo demuestra cuando el estu- } \\
\text { diante es capaz de responder libremente mediante } \\
\text { respuestas abiertas y reflexivas. }\end{array}$ & & & & & \\
\hline
\end{tabular}




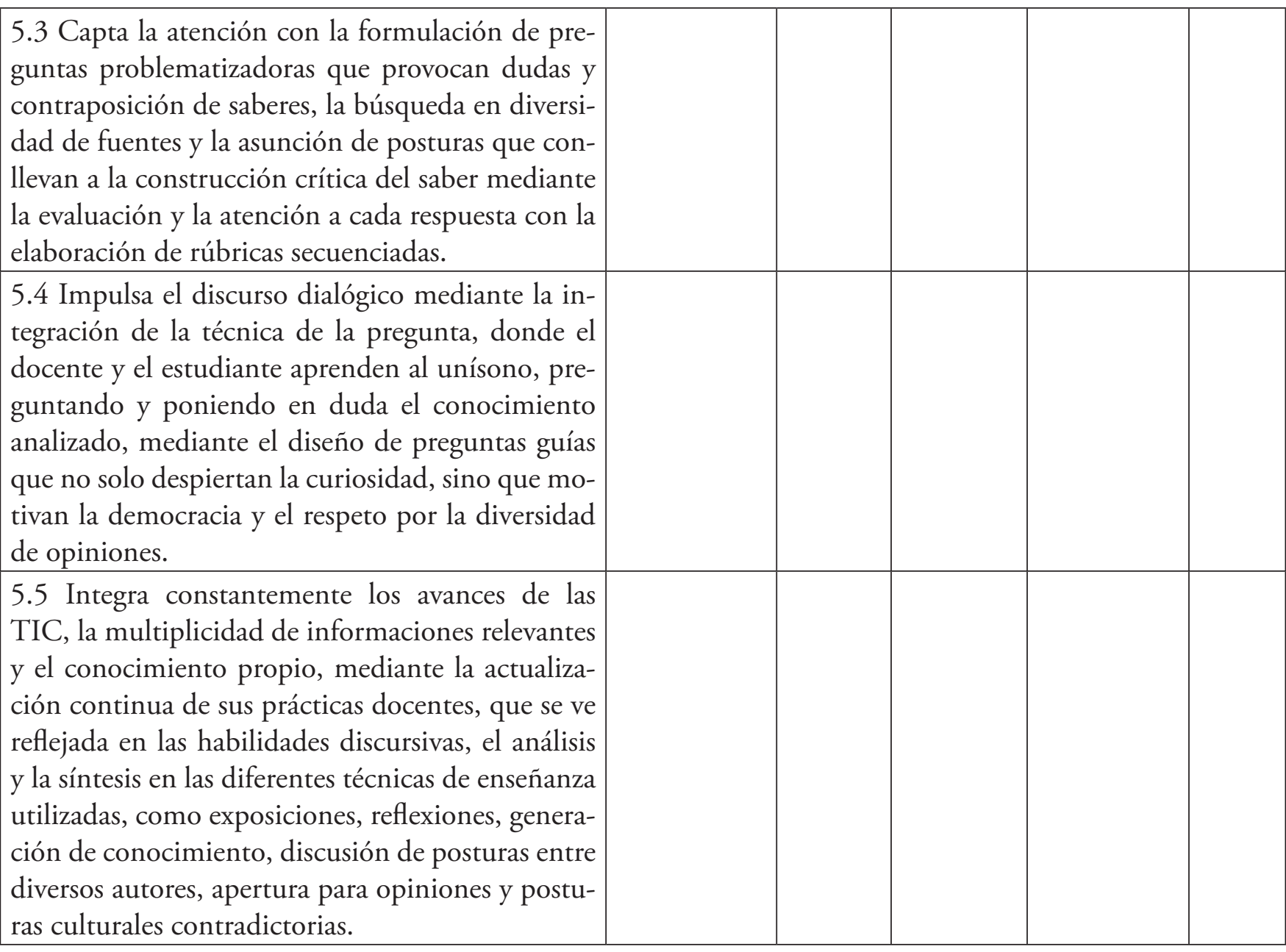

\begin{tabular}{|c|c|c|c|c|c|}
\hline VI. Comprensiva & $\begin{array}{c}\text { Excelente } \\
100-95\end{array}$ & $\begin{array}{c}\text { Bien } \\
94-85\end{array}$ & $\begin{array}{c}\text { Regular } \\
84-75\end{array}$ & $\begin{array}{c}\text { Deficiente } \\
74-65\end{array}$ & $\begin{array}{l}\text { Nulo } \\
64-0\end{array}$ \\
\hline $\begin{array}{l}\text { 6.1 Contextualiza e integra el conocimiento en } \\
\text { los diferentes ambientes en los que se desarro- } \\
\text { lla la actividad educativa, tomando como re- } \\
\text { ferencia la comunidad donde residen tanto el } \\
\text { docente, como el estudiante, mediante estudios } \\
\text { de casos dirigidos, aplicación de técnicas para la } \\
\text { recopilación de la información, como el repor- } \\
\text { taje, las entrevistas y los artículos periodísticos. }\end{array}$ & & & & & \\
\hline
\end{tabular}




\begin{tabular}{|l|l|l|l|l|}
\hline $\begin{array}{l}\text { 6.2 Vincula el tópico a tratar con circunstan- } \\
\text { cias de la realidad de vida, como son la familia, } \\
\text { el barrio, la comunidad, el contexto regional } \\
\text { y nacional. De esta forma genera preguntas y } \\
\text { situaciones de investigación - acción donde se } \\
\text { extrapolen dichas realidades, proveyendo res- } \\
\text { puestas mediante el conocimiento académico y } \\
\text { científico, con el uso de fichas y registros anec- } \\
\text { dóticos para lograr la recopilación y la vincula- } \\
\text { ción de la información. }\end{array}$ & & & & \\
\hline $\begin{array}{l}\text { 6.3 Sitúa el conocimiento en el contexto de la } \\
\text { realidad de vida del estudiante mediante la prác- } \\
\text { tica docente transdisciplinaria, en la que refleja } \\
\text { la transversalidad entre el saber y el saber hacer } \\
\text { para el planteamiento adecuado de situaciones, } \\
\text { aplicando prácticas fuera del aula, impulsando } \\
\text { campańas de servicio a los problemas sociales } \\
\text { con la comunidad y el entorno medioambien- } \\
\text { tal, promoviendo una práctica inclusiva con la } \\
\text { simulación de los juegos de roles y en prácticas } \\
\text { de campo, e impulsando la integración de los } \\
\text { diversos sectores sociales de la comunidad. }\end{array}$ & & & & \\
\hline
\end{tabular}

\begin{tabular}{|c|c|c|c|c|c|}
\hline VI. Ética & $\begin{array}{c}\text { Excelente } \\
100-95\end{array}$ & \begin{tabular}{|c|} 
Bien \\
$94-85$ \\
\end{tabular} & $\begin{array}{c}\text { Regular } \\
84-75\end{array}$ & $\begin{array}{c}\text { Deficiente } \\
74-65\end{array}$ & $\begin{array}{l}\text { Nulo } \\
64-0 \\
\end{array}$ \\
\hline $\begin{array}{l}\text { 7.1 Genera la producción de multiplicidad de co- } \\
\text { nocimientos bajo un código ético y moral que esté } \\
\text { siempre al servicio de una inteligencia colectiva, } \\
\text { sin discriminar el conocimiento empírico de los } \\
\text { sectores populares y excluidos de la sociedad, esto } \\
\text { lo manifiesta con la vinculación de los distintos } \\
\text { saberes y la generación de experiencias, partiendo } \\
\text { de la base de lo cotidiano y de la diversidad étnica } \\
\text { o social. }\end{array}$ & & & & & \\
\hline $\begin{array}{l}\text { 7.2 Demuestra actitud de respeto a la diversidad } \\
\text { de opiniones, enfatizando el derecho de autor y la } \\
\text { política de crédito, lo que queda demostrado en su } \\
\text { discurso oral y escrito. Así como en la discusión y } \\
\text { confrontación de fuentes empíricas, científicas y } \\
\text { académicas. }\end{array}$ & & & & & \\
\hline
\end{tabular}




\begin{tabular}{|l|l|l|l|l|}
\hline $\begin{array}{l}7.3 \text { Es respetuoso y tolerante con las diferentes } \\
\text { formas de aprender y de expresión de ideas por } \\
\text { parte de los alumnos; esto lo manifiesta con la in- } \\
\text { clusión de estrategias y actividades que generan el } \\
\text { aprendizaje de conocimientos que conllevan a la } \\
\text { reflexión de las distintas realidades; lo que queda } \\
\text { evidenciado con la utilización de diversas técnicas } \\
\text { de enseńanza, para captar la atención y provocar el } \\
\text { discurso dialógico. }\end{array}$ & & & & \\
\hline $\begin{array}{l}7.4 \text { Emite juicios de valor y toma decisiones refe- } \\
\text { rentes a la realidad social y cultural circundante } \\
\text { que manifiesta mediante las exposiciones, los estu- } \\
\text { dios de caso, los grupos focales, las entrevistas y la } \\
\text { extrapolación de comentarios de la cotidianidad, } \\
\text { mostrando una actitud respetuosa y reflexiva. }\end{array}$ & & & & \\
\hline $\begin{array}{l}7.5 \text { Reconoce los procesos evaluativos como un } \\
\text { tema de valoración y no de control. Lo demuestra } \\
\text { mediante el análisis y la divulgación personalizada } \\
\text { de las informaciones provenientes de dicho proce- } \\
\text { so, mostrando respeto y la intención de mejorar } \\
\text { las debilidades encontradas. Para lograrlo, sociali- } \\
\text { za con el propio alumno, sus colegas y los expertos } \\
\text { externos. }\end{array}$ & & & & \\
\hline
\end{tabular}

\section{Conclusión}

La descripción de un abordaje transdisciplinario va más allá de las experiencias externas, evocando la inclusión de elementos internos, además de su fundamentación teórica. En el presente artículo se evidencia que la práctica docente ejercida por el facilitador de la prueba piloto posee un carácter transdisciplinar, donde se entrelazan aquellas características que fueron identificadas por este estudio como necesarias: la docencia transdisciplinar es transgresora, integradora, crítica, comprensiva, inclusiva, democrática y ética; siendo con frecuencia difícil identificarlas de manera aislada, por su presencia simultánea e interrelacionada.

Las exigencias de la docencia transdisciplinaria también motivan a una reflexión en torno a los recursos que están llamados a respaldarla. Uno de ellos es el Sistema de Circulación del Conocimiento, presente en el marco teórico de esta investigación y cuyos recursos fueron dinámicamente utilizados por el docente de la experiencia de la prueba piloto. Sistema que nos plantea todas las limitaciones propias del diseño de herramientas de clasificación del conocimiento, ontologías, vocabularios, tesauros y, en general, vías de acceso a la información, diseñados y construidos desde una perspectiva unidisciplinaria.

A su vez, con esta experiencia se prueba, se motiva y se aboga por el beneficio de las prácticas transdisciplinarias en todos los contextos del saber humano y, en particular, en la práctica docente, con el propósito de generar una forma de comprensión y acercamiento del ser humano y el mundo en el que vive. 
Finalmente, todas las características propias de la docencia transdisciplinaria hacen indispensable una modalidad de evaluación propia, construida especialmente para ello, y este es un aporte que hacemos en este estudio, con la intención de que sea de valor para la comunidad académica que entiende la transdisciplinariedad como la modalidad apropiada de la docencia; esto debido a que desde el abordaje disciplinar e incluso interdisciplinar no puede darse respuesta a la complejidad del mundo actual.

\section{Bibliografía}

Bourguignon, A. (1997). De la pluridisciplinarité à la transdisciplinarité. Bulletin Interactif $d u$ Centre International de Recherches et Études Transdisciplinaires, (9-10). Recuperado de http:// ciret-transdisciplinarity.org/locarno/loca5c1.php

Caballero, R., Rascón, A., Castro, G., Rochín, F. \& Solano, M. (2014). Evaluación del desempeño docente en universidades del sur de Sonora. Revista de Investigación Académica sin Fronteras, 7(19), 1-19. Recuperado de http://revistainvestigacionacademicasinfrontera.com/inicio/wp-content/ uploads/2014/02/S19-1.pdf

Cassinari, D., Hilier, J., Miciukiewicz, K., Novy, A., Habersack, S., MacCallum, D. \& Moulaert, F. (2011). Transdisciplinary research in social polis. Bruselas: European Commission.

Espinosa, A. C. (2010). Estrategias metodológicas para operacionalizar la práctica educativa transdisciplinaria, en conjunto con los actores universitarios, en las licenciaturas del Centro de Estudios Universitarios Arkos de Puerto Vallarta, Jalisco, México (Tesis doctoral, Universidad Estatal a Distancia, San José, Costa Rica), Universidad Estatal a Distancia, San José, Costa Rica).

Galvani, P. (2007). La reflexividad sobre la experiencia: una perspectiva transdisciplinar sobre la autoformación ( $1^{\text {a }}$ parte). Visión docente con-ciencia, 6(36), 5-11.

Guzmán Gómez, M. (2008). Sistemas de organización del conocimiento y transdisciplinariedad: un acercamiento desde el enfoque de los niveles integrativos. ACIMED, 18(5). Recuperado de http:// scielo.sld.cu/scielo.php?script=sci_arttext\&pi$\mathrm{d}=$ S1024-94352008001100007

Jantsch, E. (1947). Inter- and transdisciplinary university: A systems approach to education and innovation. Higher Education Quarterly, 1(1), 7-37.

Jantsch, E. (1980). The self-organizing universe: Scientific and human implications of the emerging paradigm of evolution. Oxford: Pergamon Press.

Martínez, M. (julio 2003). Transdisciplinariedad: un enfoque para la complejidad del mundo actual. Concienciactiva, (1), 13-29.

Morín, E. (1996). Sobre la interdisciplinariedad. Sociología y Politica, 8, 17-27.

Nicolescu, B. (1998). La Transdisciplinariedad: una nueva visión del mundo. Manifiesto Centro Internacional para la Investigación Transdisciplinaria (CIRET). Paris: Ediciones Du Rocher.

Piaget, J. (1990). La equilibración de las estructuras cognitivas: problema central del desarrollo. México: Siglo XXI.

Pineau, G. (2009). Estrategia universitaria para la transdisciplinariedad y la complejidad. Visión Docente Con-Ciencia, 8(4), 2. Recuperado de http://www.ceuarkos.com/Vision_docente/revistas/48/ESTRATEGIA\%20UNIVERSITARIA.pdf

Ramírez, M. I. \& Montoya, J. (2014). La evaluación de la docencia en educación superior universitaria: una revisión de la literatura. REDU: Revista de Docencia Universitaria, Número Monográfico Dedicado a Equidad y Calidad en la Docencia Universitaria: Perspectivas Internacionales, 12(2), 77-95. 
Robalino, M. \& Korner, A. (2006). La evaluación del desempeño y carrera profesional docente. Recuperado de http://www.oei.es/evaluacion/educativa/evaluacion_desempeno_carrera_profesional_docente_unesco.pdf

Stake, R., Contreras, P. \& Arbesú, I. (2011). Evaluando la calidad de la universidad, particularmente su docencia. Perfiles educativos, 33(SPE.), 155-168.

Tejedor, F. J. T. (2012). Evaluación del desempeño docente. Revista Iberoamericana de Evaluación Educativa, 5(1), 318-327.

\section{Datos de filiación}

Mabel Marta Artidiello Moreno. Profesora investigadora. Estudió Filosofía en la Universidad de la Habana, Cuba, cursó una Maestría en Epistemología y Ciencias Sociales en la Universidad Autónoma de Santo Domingo, UASD y un Doctorado en Historia de la Filosofía en la Humboldt Universität, Berlín, Alemania. Es coordinadora de la Cátedra y de Postgrado en UASD e INTEC. Sus líneas de investigación son: Pensamiento filosófico dominicano; pensamiento creativo; guías de metodología; estudios generales; transdisciplinariedad. Correo electrónico: mabel. artidiello@intec.edu.do

María Elena Córdoba. Educadora Investigadora. Estudió Psicología en la Universidad Nacional de Córdoba, Argentina, cursó una Maestría en Educación Social y Animación Sociocultural en la Universidad de Sevilla y un doctorado en Educación en el Atlantic International University. Jorge: es en USA, pero eso es vía online. Actualmente se desempeña como asesora y consultora en Desarrollo Curricular y Formación Docente. Su línea principal de investigación está centrada en los estudios generales y la transdiciplinariedad. Correo electrónico: maria.cordoba@ intec.edu.do

Lucero Arboleda. Bibliotecóloga. Graduada en Bibliotecología en la Universidad de Antioquia, Medellín, Colombia. Realizó postgrado en Bibliotecología Biomédica, en la Universidad de Sao Paulo, Brasil; Master en Gestión Universitaria de la Universidad de Alcalá de Henares, España. Su labor de investigación bibliográfica le ha brindado la oportunidad de ser autora, coautora y coeditora de obras de referencia como: índices, bibliografías o guías metodológicas de investigación. Correo electrónico: lucero.arboleda@intec.edu.do 